\title{
Die Schenkelhalsfraktur beim Erwachsene
}

\author{
Kopfersetzende Therapie
}

\author{
Andreas Seekamp, Christine Machens, Tim Pohlemann
}

\section{Zusammenfassung}

Der Bruch des Schenkelhalses ist eine der häufigsten Frakturen des älteren Menschen. Alleine in Deutschland erleiden jährlich etwa 60000 Menschen eine solche Verletzung. Mit der Zunahme der Lebenserwartung wird die Häufigkeit der Schenkelhalsfraktur weiter steigen, da zum einen die im Alter zunehmende Osteoporose die Stabilität des Schenkelhalses vermindert und zum anderen ebenfalls im Alter auftretende kardiale und zerebrale Erkrankungen die Fallneigung verstärken. Gerade bei den älteren Patienten ist eine frühest mögliche Versorgung der Schenkelhalsfrakturen anzustreben, die eine kurze Liegedauer und eine rasche Mobilisierung unter Vollbelastung ermöglicht und dadurch zur Vermeidung vital gefährdender Immobilisationsschäden, wie z.B. einer Pneumonie, beiträgt. Unter Beachtung der Indikationen für eine primäre kopfersetzende operative
Versorgung kommen sowohl Hemials auch Totalendoprothesen in Betracht. Die Einbringung der Duokopfprothese ist ein schonendes, rasch durchzuführendes Operationsverfahren. Die Rate postoperativer Komplikationen ist gering, die Immobilisierungsphase ist kurz. Liegen zusätzlich zur Fraktur präoperative pathologische Veränderungen des Hüftgelenkes vor, sollte ein kompletter Ersatz des Hüftgelenkes (Kopf und Hüftgelenkpfanne) erwogen werden. Auch bei jüngeren Patienten kann eine kopfersetzende Therapie einer Schenkelhalsfraktur primär indiziert sein, diese sollte in der Regel durch eine Totalendoprothese erfolgen. Sekundär nach einer kopferhaltenden Versorgung auftretende Komplikationen wie Dislokation der Fraktur, Pseudarthrose oder Hüftkopfnekrose stellen ebenfalls eine Indikation für einen Gelenkersatz dar, wobei auch hier zwischen der Hemiprothese und der Totalendoprothese gewählt werden kann.

\section{Einleitung}

Die alte Dame mit schmerzverzerrtem Gesicht und außenrotiertem und verkürztem Bein ist in jeder chirurgischen Notaufnahme ein häufig zu findendes Bild. Die Schenkelhalsfraktur zählt zu den typischen Verletzungen des älteren Menschen. Der Altersgipfel liegt mittlerweile bei über 80 Jahren [8] und steigt noch. Frauen tragen eine 25\%ige, Männer eine $7 \%$ ige Chance, in ihrem Leben einen solchen Bruch zu erleiden.

OP-JOURNAL 2002; 17: 114-120

(c) Georg Thieme Verlag Stuttgart · New York
Langenbeck berichtete bereits 1878 von einem ersten Versuch, eine Schenkelhalsfraktur mittels Schraubenosteosynthese zu fixieren. „Leider zeigte sich aber nach mehreren Tagen eine Entzündung im Gelenk... Es entstand Hospitalbrand in der Schnittwunde und die Patientin ging zu Grunde“ [5].

War damals die Asepsis das grundlegende Problem, so ist man sich heute sicher, dass der wesentliche Faktor für den erfolgreichen Verlauf nach einer Schenkelhalsfraktur beim alten Menschen die frühestmögliche Versorgung und vor allem die rasche postoperative Mobilisierung ist. Es ist wiederholt erwiesen worden, dass die vital bedrohlichen pulmonalen und allgemeinmedizinischen Komplika- tionen mit der Dauer der Immobilisation exponentiell ansteigen $[1,3,7,9]$.

Ziel der Behandlung von Schenkelhalsfrakturen muss in jedem Fall die frühzeitige Mobilisierung sein.

Dieses kann in wenigen Fällen vielleicht schon durch eine konservative Behandlung erreicht werden, andernfalls stehen die kopferhaltenden Verfahren (Osteosynthese) und die kopfersetzenden Verfahren (Prothese) zur Verfügung.

\section{Diagnostik und Klassifikation}

Grundsätzlich lassen sich die Schenkelhalsfrakturen in laterale und mediale unterteilen, wobei die lateralen Schenkelhalsfrakturen deutlich seltener sind und sich von den medialen Schenkelhalsfrakturen dadurch abgrenzen lassen, dass die laterale Frakturlinie in ihrem Verlauf keine Verbindung zum Hüftkopf aufweist. Die Unterscheidung zwischen den beiden Frakturformen ist für die Wahl des Operationsverfahrens wichtig und erlaubt auch die Abschätzung der Gefahr einer posttraumatischen Hüftkopfnekrose. Letztere tritt bei den medialen Schenkelhalsfrakturen mit einer Wahrscheinlichkeit von max. 20\% bis zu zwei Jahre nach der Fraktur auf und entwickelt sich aufgrund der Fraktur begleitenden Kapselverletzung des Hüftgelenkes und der damit kompromittierten Durchblutung des Hüftkopfes.

Schon bei der klinischen Untersuchung lässt sich die Diagnose einer Schenkelhalsfraktur durch die typische Außenrotation und die Flexion sowie durch die schmerzhaft aufgehobene Beweglichkeit der betreffenden Hüfte feststellen (Abb.1).

Um eine Schenkelhalsfraktur exakt als solche erkennen und diese als mediale oder laterale identifizieren zu können, ist eine Beckenübersichts- Röntgenaufnahme erforderlich sowie eine axiale 


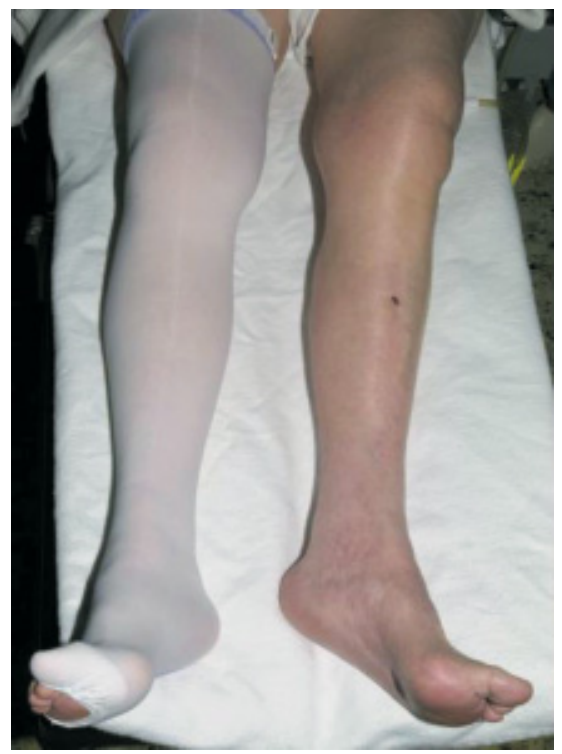

Abb. 1 Klinisches Bild einer Schenkelhalsfraktur: Charakteristisch ist eine Außenrotation und leichte Verkürzung des betroffenen Beines mit Beugung in Knie und Hüfte. Es sind jedoch nicht immer alle Merkmale zu verzeichnen.

Röntgenaufnahme der betreffenden Seite. Bei der anterior-posterioren (a.p.) Projektion ist darauf $\mathrm{zu}$ achten, dass beide Unterschenkel innenrotiert sind, um die Antetorsion des Schenkelhalses auszugleichen. Der Trochanter minor ist bei richtiger Projektion des Schenkelhalses in der a. p. Aufnahme nicht zu sehen. Zur Durchführung der axialen Aufnahme wird die Röntgenkassette lateral der verletzten Seite am Hüftgelenk angestellt. Das gesunde Bein der Gegenseite wird in der Hüfte auf $90^{\circ}$ gebeugt und angehoben, die Röntgenröhre wird anschließend von der gesunden Seite mit Ziel auf die verletzte Hüfte eingestellt. Der Röntgenstrahl trifft so den frakturierten Schenkelhals durch den Schritt direkt axial (Abb. 2).

Die Klassifikation von medialen Schenkelhalsfrakturen kann nach zwei verschiedenen Einteilungen vorgenommen werden. Die Klassifikation nach Pauwels berücksichtigt im Wesentlichen den Winkel der Frakturlinie zur Horizontalebene (Abb.3). Je senkrechter die Frakturlinie verläuft, desto höher wird die Gefahr einer sekundär weiter fortschreitenden Dislokation unter der Mobilisierung eingeschätzt und desto zwingender wird eine operative Versorgung für erforderlich erachtet.

Ebenfalls weit verbreitet ist die Klassifikation nach Garden (Abb.4). Hier wird

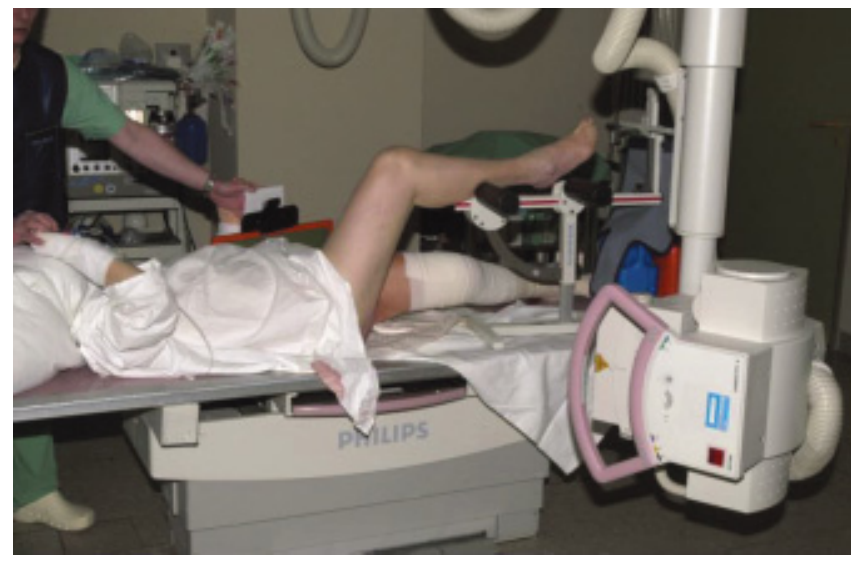

Abb. 2 Röntgeneinstellung bei einer axialen Hüftaufnahme: Die Filmkassette wird an der verletzten Seite angestellt, die Röntgenröhre wird von der Gegenseite bei angehobenem Bein auf die Kassette zentriert.

in erster Linie der Dislokationsgrad der Fraktur berücksichtigt. Ca. $10-30 \%$ der medialen Frakturen stehen nicht oder nur wenig verschoben oder sind in eine leichte Valgusstellung impaktiert (Klassifizierung nach Garden Grad I-II) [1]. In diesen Fällen kann ein konservativer Therapieversuch mit ca. $15 \mathrm{~kg}$ Teilbelastung des verletzten Beines unternommen werden. Dabei kann es jedoch zu starken Anteversionsfehlstellungen durch eine sekundäre Verkippung der Fraktur (10$27 \%$ ) kommen, so dass häufig doch eine operative Versorgung nötig wird $[1,9]$. Bei den wesentlich häufigeren dislozierten Brüchen (Garden III - IV) ist von vorne herein eine operative Versorgung indiziert. Laterale Schenkelhalsfrakturen bedürfen immer der operativen Versorgung.

Für die operative Stabilisierung von medialen und lateralen Schenkelhalsfrakturen stehen grundsätzlich die verschiedenen kopferhaltenden Verfahren und die kopfersetzenden Verfahren zur Verfügung. Auf das letztere Verfahren wird hier näher eingegangen.

\section{Indikationen für die kopfersetzende Therapie}

Schenkelhalsfrakturen sollten nicht grundsätzlich durch eine Prothese versorgt werden.

Insbesondere bei den jüngeren und körperlich noch aktiven Patienten sollte primär eine kopferhaltende Therapie angestrebt werden. Vorrausetzung ist natürlich, dass keine begleitende Schädigung des Hüftkopfes vorliegt. Der Einsatz von Endoprothesen scheint nach der Literatur beim alten, osteoporotischen oder vorgeschädigten Gelenk die Therapie der Wahl $[1,3,7]$. Zumal dieses Verfahren nach einer prospektiven Untersuchung $12 \mathrm{Mo-}$ nate nach der Versorgung in 90\% der Fälle zu einer kompletten Schmerzfreiheit geführt hatte und $85 \%$ der Patienten mit Totalendoprothese wieder ohne Gehhilfe gehen konnten [10].

Die Entscheidung eine Schenkelhalsfraktur kopferhaltend oder kopfersetzend zu behandeln, sollte sich letzten Endes individuell zum einen nach dem Frakturtyp und zum anderen nach dem prä-operativen Allgemein- und Mobilisierungszustand des Patienten richten.

Unter diesen Gesichtspunkten lassen sich folgende Indikationen für die primäre kopfersetzende Therapie einer Schenkelhalsfraktur herausstellen:

- Frakturen der Klassifikation Garden III und IV (gilt für die mediale Fraktur)

- Frakturen, die erst nach deutlich mehr als 6 Stunden nach dem Ereignis versorgt werden können (gilt für die mediale Fraktur)

- Frakturen die sich nicht anatomisch reponieren lassen (mediale wie laterale)

- Frakturen bei vorbestehender Degeneration des betreffendes Gelenkes

- begleitende Frakturen des Hüftkopfes

- die pathologische Fraktur

Das kopferhaltende Verfahren ist zeitkritisch und technisch anspruchsvoll. Eine anatomische Reposition mit anschließender Fixierung ist notwendig, da es sonst zu einer Minderversorgung des Hüftkopfes und somit zur Hüftkopfnekrose kommen kann. Diese Minderversorgung entsteht durch die Läsion der A. circumflexa femoris medialis und ihrer Äste sowie der A. rami ascendens arteriae femoris lateralis, welche durch den Druck des Frakturhämatoms und durch die mechanische Knickung im Bereich der Fraktur kompromittiert sind. Die Angaben zur Häufigkeit der Hüftkopfnekrose schwanken in der Literatur zwischen 0 
und 30\% $[4,7,9]$. Die Versorgung der Fraktur innerhalb von 6 Stunden nach Trauma reduziert die Nekroserate signifikant. Die Inzidenz ist des Weiteren abhängig vom Alter des Patienten, von den Vorerkrankungen, vom Dislokationsgrad der Fraktur, der Repositionsgüte und der Art der Osteosynthese $[4,9]$.

Gelingt die kopferhaltende Frakturversorgung nicht innerhalb von 6 Stunden nach dem Trauma ist daher eine primäre prothetische Versorgung in Betracht zu ziehen.

Neben den frakturbedingten Indikationen kann auch von Seiten des Allgemeinzustandes des Patienten eine primäre prothetische Frakturversorgung indiziert sein, wenn der Patient z.B. nicht in der Lage ist, eine Teilbelastung durchzuführen. Der Zustand des Patienten ist häufig derart, dass eine Narkose besser toleriert wird als eine langfristige Bettlägerigkeit oder eine sehr verzögerte Mobilisierung. Daher bietet die operative und damit ad hoc belastungsstabile Versorgung der Fraktur durch eine Prothese auch für den Patienten im schlechten Allgemeinzustand den Vorteil der frühen Mobilisierung, auch wenn der operative Eingriff etwas invasiver ist als z.B. eine einfache Schraubenosteosynthese.

Die kopfersetzende Operation kann auch als sekundäre Versorgung nach einer primär kopferhaltenden Therapie einer Schenkelhalsfraktur notwendig werden:

- sekundäre Frakturdislokation nach primärer Osteosynthese

- sekundäre Frakturdislokation nach primär konservativer Behandlung und der Feststellung eines der oben genannten Kriterien für die primär kopfersetzende Therapie, andernfalls ist in diesem speziellen Fall auch die sekundäre Osteosynthese gerechtfertigt
- die nicht verheilte Fraktur

- die posttraumatische Hüftkopfnekrose

- die fortschreitende Koxarthrose (nicht unmittelbare Folge der Fraktur)

\section{Methoden der kopfersetzenden Versorgung}

Bei den kopfersetzenden Verfahren nach Schenkelhalsfaktur gibt es im Wesentlichen zwei unterschiedliche Prothesenarten und zwei unterschiedliche Verankerungsmöglichkeiten. Man unterscheidet zwischen Hemi- und Totalarthroprothesen und zwischen zementierten und unzementierten Verfahren.

Als Beispiel einer Hemiprothese, nur kopfersetzend, sei die bipolare oder Duokopfprothese genannt. Diese besteht aus einem Schaft, der in das Femur eingebracht und zumeist mit Zement fixiert wird. Auf dessen Hals wird ein Kopf gesetzt, der aus einer Metallkugel besteht, die in einer Kunststoffschale liegt, die sich ihrerseits in einer Metallfassung befindet. Diese Metallschale, welche den großen Kopf repräsentiert und etwa dem Durchmesser des resezierten Femurkopfes entsprechen sollte, artikuliert dann in der unversehrt belassenen Hüftgelenkspfanne. Alle drei Komponenten sind dabei ineinander beweglich. Damit wird die Kraftübertragung aus der Risikozone zwischen Prothesenkopf und Azetabulum in das neue, zweite Gelenk zwischen Metallkugel und Kunststoffschale verlegt [4]. Prothesen mit einem dem Schaft bereits fest aufsitzenden Kopf sind weitgehend verlassen worden. In der Regel wird heute ein modulares System verwendet. Die Bohrung am Prothesenkopf, in welche der Prothesenschaft hineinpasst, ist unterschiedlich tief ausgeprägt und erlaubt somit eine gewisse Längendifferenzierung bei der Prothesenimplantation (Abb.5).
Beim totalendoprothetischen Ersatz (TEP) wird neben dem frakturierten Schenkelhals samt Kopf auch die Hüftpfanne ersetzt. Der Gelenkknorpel wird hierbei komplett herausgefräst bis man auf spongiösen Knochen gerät. Die eingesetzte Gelenkpfanne besteht entweder aus einem metallischen Gerüst, welches dann die Kunststoffschale für den Prothesenkopf aufnimmt, oder sie besteht nur aus Kunststoff. Letzteres Modell wird nur verwendet, wenn die Pfanne gleich einzementiert wird. Die heute jedoch häufiger verwendete Methode besteht in der zementfreien Fixation der Pfanne. Hierbei wird das metallische Gerüst entweder durch einen Spreizmechanismus oder durch Schrauben im Azetabulum fixiert. Anschließend wird das Kunststoffinlay in die Metallpfanne eingeschraubt oder eingeklemmt (Abb.6 u. 7).

Die Entscheidung, eine Totalendoprothese (TEP) oder eine Duokopfprothese zu implantieren sollte unter Berücksichtigung folgender Aspekte erfolgen. Aufgrund der strafferen Führung des Prothesenkopfes in der Pfanne geht man davon aus, dass die TEP eine längere Lebensdauer im Vergleich zur Duokopfprothese hat. Bewiesen ist dieses jedoch bisher nicht, da die Mehrzahl der implantierten Duokopfprothesen bisher die Patienten überlebt hat. Dennoch sollte man sich bei den jüngeren Patienten eher für die Implantation einer TEP entscheiden. Die Verwendung einer Totalendoprothese ist auch indiziert, wenn neben der Fraktur noch pathologische Veränderungen im Pfannenbereich bestehen, wie beispielsweise eine Koxarthrose, eine Dysplasie, oder eine systemische Grunderkrankung wie eine Polyarthritis, welche das Hüftgelenk mitbetrifft. Wegen der Präparation der Hüftgelenkspfanne ist die Operationszeit einer TEP im Vergleich zur Duokopfprothese jedoch um etwa ein Drittel
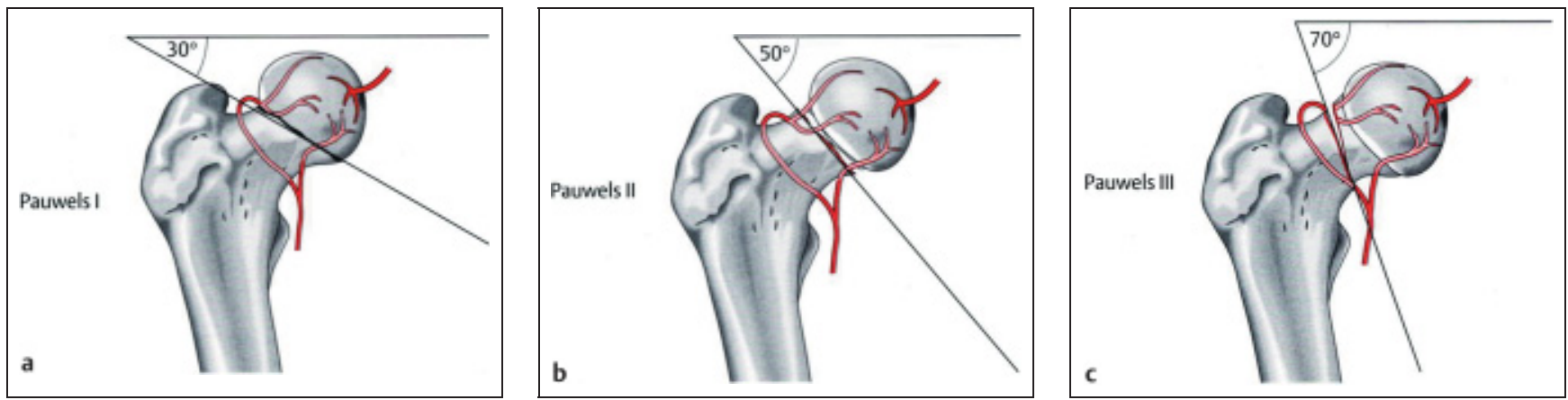

Abb. 3a-c Klassifikation der medialen Schenkelhalsfraktur nach Pauwels: Für die Klassifizierung ist der Frakturverlauf zur Horizontalebene entscheidend (in Anlehnung an: „Praxis der Unfallchirurgie“, Ed: W. Mutschler, N. Haas, Thieme Verlag Stuttgart, 1999). 


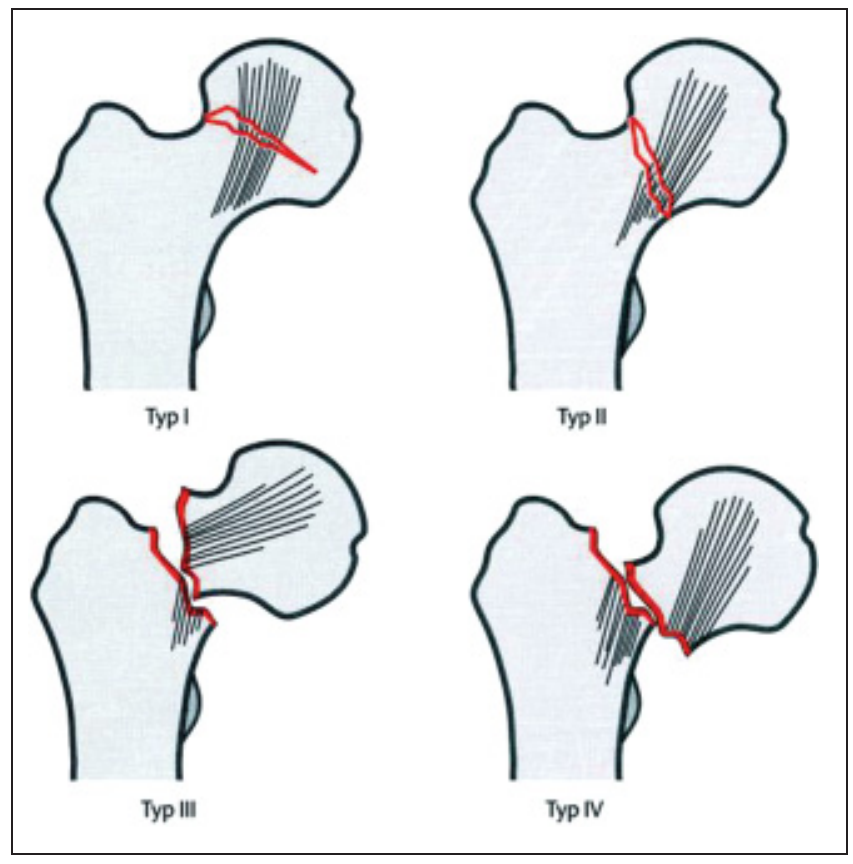

Abb. 4 Klassifikation der medialen Schenkelhalsfraktur nach Garden: Diese Einteilung richtet sich nach dem primären Dislokationsgrad der Fraktur (in Anlehnung an: „Praxis der Unfallchirurgie", Ed:

W. Mutschler, N. Haas, Thieme Verlag Stuttgart, 1999).
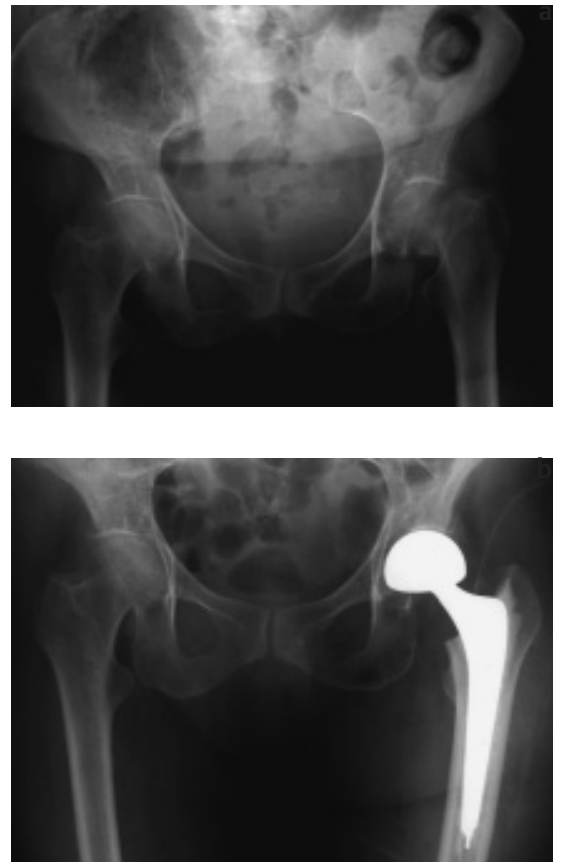

Abb. 5a u.b Röntgen einer Schenkelhalsfraktur (a), die primär mit einer Duokopfprothese versorgt wurde (b).

länger und der Blutverlust ist wegen der großen ausgefrästen Spongiosafläche des Azetabulums signifikant höher. Aus diesen Gründen ist die Verwendung einer Duokopfprothese bei dem Patienten in schlechtem Allgemeinzustand in jedem Fall der Implantation einer Totalendoprothese vorzuziehen. Sind mehrere Faktoren sowohl für die eine als auch für die andere Methode in einem Patienten zu berücksichtigen, ist eine individuell an-

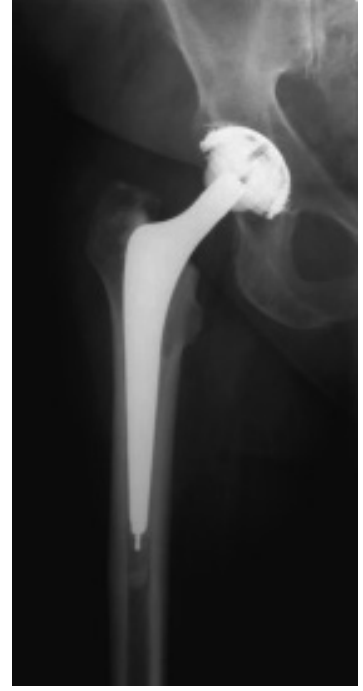

Abb. 6 Röntgen einer Schenkelhalsfraktur nach Versorgung mit einer Totalendoprothese: Zementfreie Pfanne mit Spreizverankerung und zementierter Schaft.

gepasste Nutzen- Risikoabschätzung vorzunehmen.

Die Frage der Fixierung der Prothese kann für die Pfanne als klar entschieden angesehen werden. Nach mittlerweile langjährigen guten Erfahrungen mit den unterschiedlichsten zementfreien Fixierungsmöglicheiten von metallischen Pfannengrundgerüsten besteht kaum mehr eine Indikation für eine zemen- tierte Pfannenfixierung. Anders verhält es sich bei dem Prothesenschaft. Dieser wird nach wie vor entweder unzementiert oder zementiert eingebracht. Der Vorteil des Zementes ist die bessere Anpassung des vorgefertigten Metallschaftes an die individuelle Femurstruktur. Als Nachteil haben sich systemische, kardiopulmonale Reaktionen beim Einbringen des Zementes (Hypotonie, Hypoxämie) und die höhere Rate an Spätkomplikationen wie periprothetische Frakturen und Implantatlockerungen ergeben. Die Operationsdauer nimmt mit der Verwendung von Zement zu, auch der Blutverlust steigt. Zudem wirken sich die Temperaturen von bis $\mathrm{zu} 70^{\circ} \mathrm{C}$, die beim Aushärten des Zementes entstehen, schädigend auf die Knochenstruktur aus. Bei jüngeren Patienten, bei denen primär wegen der Fraktur oder sekundär, z.B. wegen einer Hüftkopfnekrose, die Implantation einer Prothese erfolgt, wird gerne auf eine Zementierung des Schaftes verzichtet, da eine gegebenenfalls anstehende Wechseloperation nach Zementierung wesentlich schwieriger und komplikationsträchtiger ist.

Allerdings birgt auch die unzementierte Methode Gefahren in sich, da es bei dem Versuch, die Prothese besonders sicher und fest einzubringen, zu einem signifikant höheren Auftreten von Femurschaftfrakturen und Schaftpenetrationen kommt. Außerdem konnte in verschiedenen Studien eine erhöhte Rate an Revisionen nach unzementierten Duokopfprothesen nachgewiesen werden, was auf die Rotation und die daraus resultierende Fehlstellung des implantierten Schaftes im intramedullären Kanal zurückgeführt wurde [2].

Die Verwendung von Zement wiederum scheint sich positiv auf die postoperative Schmerzintensität und deren Dauer auszuwirken. Auch die Mobilität im ersten Jahr nach Operation war in einigen Studien gegenüber den unzementiert eingebrachten Hemiprothesen verbessert. Andererseits gibt es Hinweise, dass die Rate der heterotopen Ossifikationen in der Gruppe der zementierten Schäfte höher ist.

\section{Hinweise zur operativen Technik}

Nach der Entscheidung über die Art der kopfersetzenden Operation stellt sich als nächstes die Frage, wie der Patient zu lagern ist. 


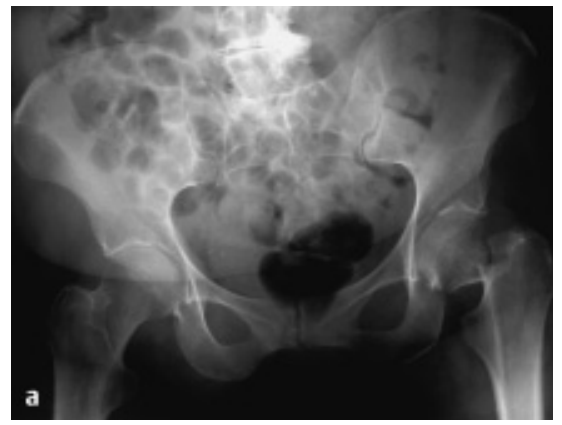

Abb.7a-d Röntgen einer medialen Schenkelhalsfraktur (a), die primär mit einer nicht einwandfreien Verschraubung nach Manninger behandelt wurde (b). Es kam zur sekundären Dislokation (c), die anschließend durch eine Prothese versorgt wurde (d).
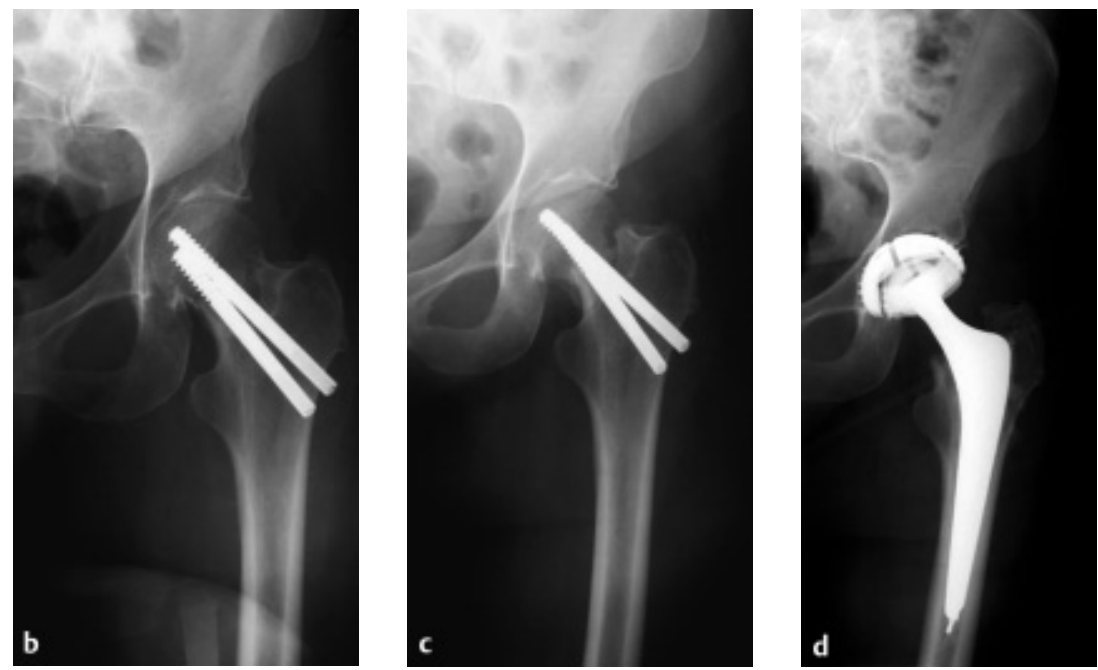

Abb. 8 Seitenlagerung einer Patientin mit steriler Abdeckung zur Versorgung einer Schenkelhalsfraktur mit Prothese.

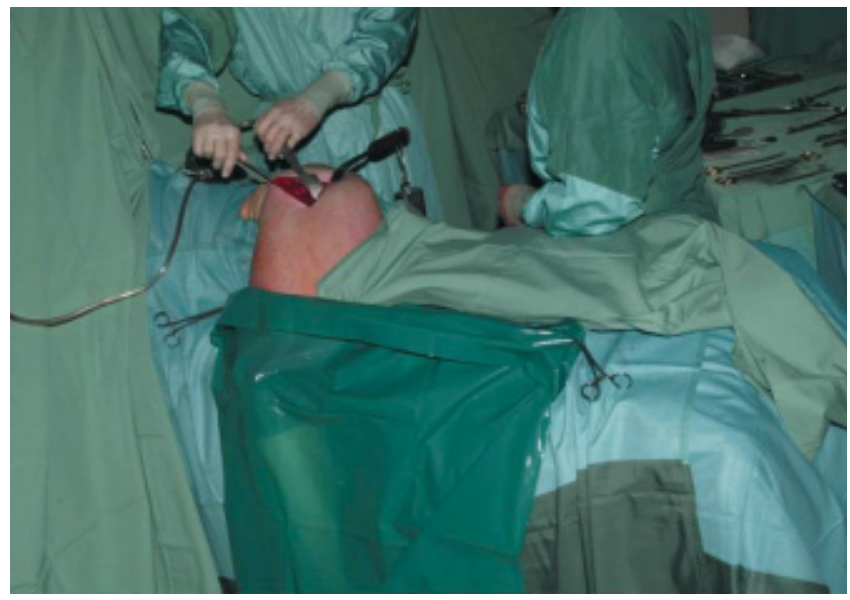

beugung über die dem Operateur gegenüberliegende Tischkante in einem sterilen Tuch abgelegt (Abb.9).

Als operativer Zugang kann entweder der transgluteale Zugang nach Bauer (modifizierter antero-lateraler Zugang zum Hüftgelenk) gewählt werden oder der dorsale Zugang. Beide Verfahren sind als gleichwertig $\mathrm{zu}$ betrachten. Beim $\mathrm{Zu}-$ gang nach Bauer wird das Hüftgelenk nach vorne durch Außenrotation des Beines luxiert, während beim dorsalen Zugang das Hüftgelenk durch Innenrotation luxiert wird.

Abb. 9 Seitenlagerung einer Patientin mit Abdeckung und intraoperativ übergelagertem Bein zur Implantation des Prothesenschaftes.
Prinzipiell kann eine Hüftgelenksersatzoperation sowohl in Rückenlage als auch in Seitenlage vorgenommen werden.

Die Seitenlage (Abb. 8) bietet dem Operateur und allen an der Operation beteiligten Assistenten einen sehr guten Einblick in das Operationsfeld. Dieses ist bei Rü- ckenlage des Patienten nicht immer gegeben. Andererseits wird die Rückenlage besser von dem Patienten toleriert und von Seiten der Anästhesie wird man eher zu einer periduralen anstatt einer ITN-Narkose geneigt sein. Im eigenen Vorgehen wird die Seitenlagerung bevorzugt, das Bein wird nach Luxation des Hüftgelenkes zur Präparation des Schaftes in $90^{\circ}$ Außenrotation und $90^{\circ}$ Hüft-
Sowohl das Pflegepersonal als auch der Patient sind über den operativen Zugang aufzuklären, da hierdurch auch festgelegt ist, welche Bewegung der Patient postoperativ vermeiden muss, um nicht eine Luxation der Prothese zu provozieren.

Nach Darstellung der Hüftgelenkkapsel wird diese inzidiert und anschließend nur bei Implantation einer Totalendoprothese komplett bis auf den Pfannenrand reseziert. Bei Implantation einer Duokopfprothese wird die Kapsel nicht entfernt, sondern die Ränder werden jeweils mit einer Haltenaht gesichert. Nach Reposition des implantierten Duokopfes wird die Kapsel dann wieder vernäht.

Nach Eröffnung des Gelenkes wird unabhängig von der Art der Fraktur zunächst eine Osteotomie im lateralen Bereich des Schenkelhalses derart durchgeführt, dass der Femurschaft für die Aufnahme des Prothesenschaftes vorbereitet werden kann. Mit der Osteotomie sollte ein Abstand von mindestens $1 \mathrm{~cm}$ zum Trochanter minor bewahrt werden. Erst hiernach wird der frakturierte Schenkelhals 
inklusive des Femurkopfes reseziert. Im Weiteren wird dann bei Verwendung einer Totalendoprothese das Azetabulum aufgefräst und die Pfanne implantiert. Anschließend wird sich dem Femurschaft zugewendet, der entsprechend aufgeraspelt wird. Bei Implantation der Duokopfprothese kann sich nach Resektion des Femurkopfes gleich diesem Schritt zugewendet werden.

Wird der Prothesenschaft durch Zement fixiert, sollte darauf geachtet werden, dass eine antibiotikahaltige Mischung verwendet wird. Das Ausweichen des Zementes bis in das distale Ende des Femurmarkraumes ist durch Einbringen einer so genannten Markraumsperre zu vermeiden. Diese kann aus einer einfachen Spongiosaplombe bestehen, die in Abhängigkeit der Prothesenschaftlänge in den Markraum vorgeschoben wird. Der Zement sollte nach Möglichkeit unter Vakuum angerührt werden. Während der Injektion des Zementes und dem Einsetzen des Prothesenschaftes in den Femur muss die Markhöhle entlüftet werden. Dies kann entweder durch ein distal am Femur angelegtes Bohrloch erfolgen, oder durch einen kleinen Entlüftungsschlauch, welcher bis kurz vor dem endgültigen Festschlagen des Prothesenschaftes im Markraum belassen wird.

Bei der zementfreien Implantation einer Pfanne und des Schaftes ist auf eine exakte Passform der gewählten Prothesengröße zu achten. Unabhängig von der Art der Fixierung sollte die Pfanne in $45^{\circ}$-Kippung und etwa $10^{\circ}$-Antetorsion eingebracht werden, der Schaft sollte ebenfalls unter einer Antetorsion von etwa $10^{\circ}$ implantiert werden. Der Prothesenschaft sollte soweit eingeschlagen werden, bis die Spitze des Prothesenhalses in einer Ebene mit der Spitze des Trochanter major zu liegen kommt. Diese Ebene wiederum muss senkrecht auf der Femurschaftachse stehen (Abb.10). Anschließend muss nach Probereposition unter Verwendung eines Probekopfes die Prothese auf ihre freie Beweglichkeit und eine ggf. bestehende Luxationstendenz geprüft werden. Unter Umständen ist ein vom Probekopf abweichender wahrer Prothesenkopf zu implantieren.

Postoperativ kann der Patient mit einem zementierten Prothesenschaft in Abhängigkeit von den Wundschmerzen unter voller Belastung mobilisiert werden. Dies wird in erster Linie für Patienten mit einer Duokopfprothese zutreffen. Patienten mit einer zementfreien Prothese

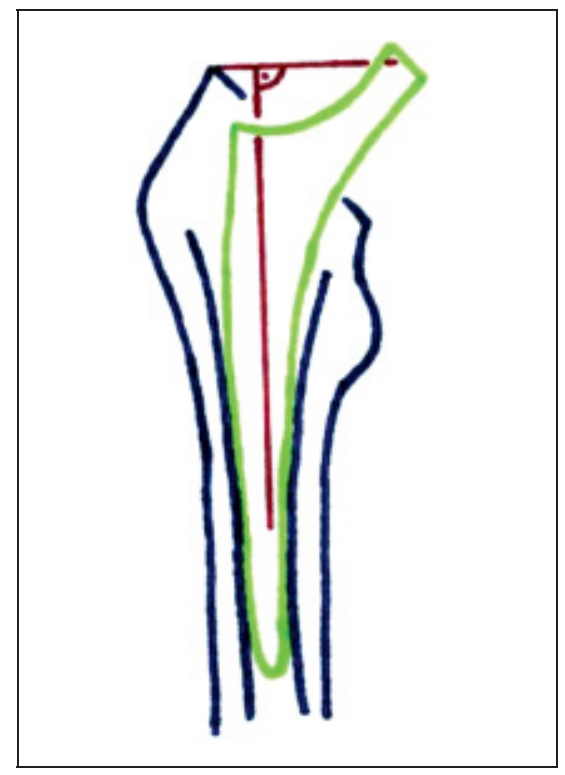

Abb.10 Intraoperatives Ausrichten des Prothesenschaftes gegenüber dem Trochanter major: Die Spitze des Prothesenhalses sollte in einer Ebene mit dem Trochanter major stehen. Die Ebene selbst muss im rechten Winkel zur Femurschaftachse liegen.

müssen eine Teilbelastung von mindestens 6 Wochen einhalten, um ein Einheilen des Prothesenschaftes ohne weiteres Nachsintern zu ermöglichen.

\section{Komplikationen}

Sowohl intra- als auch postoperativ können sich unterschiedlichste Komplikationen ergeben. Bei der Einbringung von Prothesen ereignen sich Fissuren, Perforationen oder gar Sprengungen des Femurschaftes. Auch Gefäß- und Nervenverletzungen können auftreten. Die systemischen Auswirkungen bei Verwendung von Zement wurden bereits weiter oben beschrieben. Es kann zu unbeabsichtigten Beinlängendifferenzen und Rotationsfehlstellungen kommen. Postoperativ besteht eine erhöhte Gefahr von Thrombosen und Embolien sowie Wundheilungsstörungen, Infekten, Serom- und Hämatomausbildung. Die Luxation der Endoprothese ist eine ebenso typische Komplikation (um 11\%) [10]. Gegenüber Totalendoprothesen, die wegen Koxarthrose eingebracht wurden, luxieren die nach Trauma verwendeten Prothesen häufiger. Vermutlich weil die Mobilität im Gelenk und der umgebenen Muskulatur präoperativ deutlich besser war als bei den arthrotisch veränderten Gelenken [9].
In einer Metaanalyse [6] zeigte sich, dass die Revisionsrate innerhalb der ersten zwei Jahre nach einer Totalendoprothese zwischen $6 \%$ und $18 \%$ liegt, hingegen zwischen $20 \%$ und $36 \%$ nach kopferhaltender Therapie.

Typische Spätkomplikation ist auch die Ausbildung heterotoper Ossifikationen, der man mit Antiphlogistikagabe und Bestrahlung beizukommen bemüht ist [2].

Kommt es zur Lockerung der Implantate oder gar im Rahmen eines Traumas zu periprothetischen Frakturen, ist eine ReOperation nötig. Bei der Verwendung von Hemiprothesen kann es außerdem zum Nachlassen der Hüftpfanne und damit zur Verschiebung der Pfannenebene in Richtung auf das kleine Becken kommen. Diese Protrusionen sind mit starken Schmerzen und einer Einschränkung der Beweglichkeit verbunden und bedürfen der operativen Revision.

\section{Schlussfolgerung}

Die frühe Mobilisierung ist die wesentliche Voraussetzung für eine erfolgreiche Versorgung von Schenkelhalsfrakturen beim älteren Menschen.

Für die Versorgung der Fraktur muss daher eine belastungsstabile Methode gewählt werden. Nicht die Fraktur selbst ist für die Patienten lebensbedrohend, sondern es sind häufig schwer wiegende Begleiterkrankungen, meist auf internistischem Fachgebiet, die nicht selten die Ursache des Sturzes und damit der Fraktur darstellen und auch den weiteren Verlauf wesentlich mitbeeinträchtigen. Die Beeinträchtigung des vielleicht ohnehin schon schlechten Gesamtzustandes durch die Schenkelhalsfraktur sollte möglichst gering gehalten werden.

Die konservative Behandlung nicht dislozierter Schenkelhalsfrakturen und die osteosynthetische, kopferhaltende Versorgung setzen voraus, dass es dem Patienten möglich ist, über einen längeren Zeitraum eine Teilbelastung des Beines einzuhalten. Sowohl die mentalen wie auch die physischen Voraussetzungen zur Verwendung von Unterarmgehstützen müssen dafür vorhanden sein. Da die Schenkelhalsfraktur vornehmlich ein Bruch des alten Menschen ist, sind diese Voraussetzungen oft nicht erfüllt. In diesen Fällen sollte eine initiale Versorgung angestrebt werden, die eine sofortige Vollbelastung der verletzten Extremi- 
tät und Mobilisierung des Patienten ermöglicht.

Hierzu stehen Hemi- und Totalendoprothesen zur Verfügung.

\section{Literatur}

${ }^{1}$ Beck A, Rüter A. Therapiekonzepte bei Schenkelhalsfrakturen. Teil 2. Chirurg 2000; 71: 347-354

${ }^{2}$ Khan RJK, MacDowell A, Crossman P, Keene GS. Cemented or uncemented hemiarthroplasty for displaced intracapsular fractures of the hip - a systemic review. Injury 2002; 33: 13-17

${ }^{3}$ Kinzl L, Bischoff M, Beck A. Endoprothesenversorgung bei medialer Schenkelhalsfraktur. Chirurg 2001; 72: 1266-1270

${ }^{4}$ Kutscha-Lissberg F, Schildhauer TA, Kollig E, Muhr G. Proximale Femurfrakturen: Wann Osteosynthese, wann Endoprothese? Die
Osteosynthese der medialen Schenkelhalsfraktur. Chirurg 2001; 71: 1253-1265

${ }^{5}$ von Langenbeck B. Verhandlungen der Deutschen Gesellschaft für Chirurgie. 1878; 7: 92

${ }^{6}$ Lu-Yao GL, Keller RB, Littenberg B, Wennberg JE. Outcomes after displaced fractures of the femoral neck. A meta-analysis of one hundred and six published reports. JBJS 1994; 76: $15-25$

${ }^{7}$ Möllenhoff G, Walz M, Clasbrummel B, Muhr G. Schenkelhalsbruch; Osteosynthese oder welche Endoprothese ist indiziert? Orthopäde 2000; 29: 288-293

8 Parker MJ. The Management of Intracapsular Fractures of the Proximal Femur. J Bone Joint Surg Br 2000; 82 B: 937-941

${ }^{9}$ Schmidt AH, Swiontkowski MF. Femoral Neck Fractures. Orthop Clin North Am 2002; 33(1): 97-111

${ }^{10}$ Skinner P, Riley D, Ellerly J, Beaumont A, Coumine R, Shafighian B. Displaced subcapital fractures of the femur: a prospective randomised comparison of internal fixation, hemiarthroplasty and total hip replacement. Injury 1989; 20: 291-293
Prof. Dr. med. Andreas Seekamp Ltd. Oberarzt der Klinik

Dr. med. Christine Machens

Assistenzärztin

Prof. Dr. med. Tim Pohlemann

Direktor der Klinik

Universitätsklinik des Saarlandes Klinik für Unfall-, Hand- und Wiederherstellungschirurgie

Kirrberger Str. 1

D-66421 Homburg/Saar 